\title{
EDITORIAL
}

\section{IgG4-related disease: why high lgG4 and fibrosis?}

\author{
Takao Koike* \\ See related research by Tsuboi et al., http://arthritis-research.com/content/14/4/R171
}

\begin{abstract}
The hallmarks of IgG4-related disease (IgG4-RD) are lymphoplasmacytic tissue infiltration with a predominance of IgG4-positive plasma cells, accompanied by fibrosis, obliterative phlebitis, dacryoadenitis, and elevated levels of IgG4. In a recent issue of Arthritis Research \& Therapy, Tsuboi and colleagues demonstrated that regulatory $T$ (Treg) celland Thelper 2 (Th2) cell-derived cytokines contribute to the pathogenesis of Mikulicz's disease, an activation pathway that appears to be common for lgG4-RD. Additional organ-specific factors may account for the different organ involvement of IgG4-RD.
\end{abstract}

IgG4-related disease (IgG4-RD) is a newly categorized disease entity initially recognized in Japan but increasingly also in other parts of the world [1,2]. Most often the diagnosis is made in patients with autoimmune pancreatitis. Additional presentations include patients with lacrimal and salivary gland involvement, formerly Mikulicz's disease (MD), which was once thought to be a subset of Sjögren's syndrome (SS).

The hallmarks of IgG4-RD are lymphoplasmacytic tissue infiltration with a dominance of IgG4-positive plasma cells, accompanied by fibrosis, obliterative phlebitis, dacryoadenitis, and elevated levels of IgG4. The pathogenesis of IgG4-RD is poorly understood; findings consistent with both an autoimmune disorder and an allergic disorder are present $[1,2]$.

IgG4 production is controlled primarily by $\mathrm{T}$ helper 2 (Th2) cells $[3,4]$. Th2 cytokines interleukin-4 (IL-4) and IL-13 enhance the production of IgG4 and IgE. In contrast, IL-10, IL-12, and IL-21 shift the balance between IgG4 and IgE, favoring IgG4. In the Th2 cytokine-driven immune reaction, IgG4 production is induced preferentially by the activation of IL-10 produced by regulatory $\mathrm{T}$

*Correspondence: tkoike@med.hokudai.ac.jp

Graduate School of Medicine, Hokkaido University, Sapporo, N-15 W-7, Kita-ku, Sapporo, Japan 060-8638
(Treg) cells [3]. Thus, selective IgG4 induction is referred to as the combined effect of Th2 and Treg cells.

In a recent issue of Arthritis Research \& Therapy, Tsuboi and colleagues [5] analyzed the expression of IgG4-specific class switch molecules such as Th2 cytokines (IL-4 and IL-13) and Treg cytokines (IL-10 and TGF- $\beta$ ), IgG4-nonspecific B cell regulatory molecules (CD40, CD154, BAFF, APRIL, and IRF4), and activationinduced cytidine deaminase (AID) in the labial salivary glands (LSGs) and peripheral blood mononuclear cells (PBMCs) from patients with IgG4-RD (MD) and SS. The authors provided evidence that IL-10, TGF- $\beta$, and AID were overexpressed in LSGs from IgG4-RD (MD) compared with those in patients with SS, suggesting that Treg cytokines (IL-10 and TGF- $\beta$ ) contribute to IgG4-specifc class switch recombination and fibrosis in patients with IgG4-RD (MD) in combination with the IgG4-unrelated molecule, AID (Figure 1).

Very recently, Tanaka and colleagues [6] examined the Th1, Th2, and Treg cytokine expression in LSGs from patients with IgG4-RD and SS. The authors showed that the levels of mRNA for both Th2 and Treg cytokines were significantly higher in LSGs from patients with IgG4-RD (MD) but that the expressions of Th1 and Th2 cytokines were higher in LSGs from patients with SS. The upregulation of Treg cytokines is identical to the findings reported by Tsuboi and colleagues [5], indicating that Treg cells play an important role in the pathogenesis of IgG4-RD (MD). In contrast, Tsuboi and colleagues showed that Th2 cytokines such as IL-4 and IL-13 were not significantly overexpressed in LSGs from patients with IgG4-RD (MD) but were increased if compared with those in healthy subjects. This finding supports the notion that Th2 cytokines such as IL- 4 and IL-13 play a common B cell-activating role in both IgG4-RD (MD) and SS. Contrary to Th2 and Treg cytokines, Th1 cytokines were upregulated only in LSGs from patients with SS [6], suggesting that Th1 cells function as key players in the pathogenesis of SS.

Consistent with the findings in MD, analyses of the expression of cytokines in inflammatory lesions from patients with IgG4-related sclerosing pancreatitis and cholangitis [7] or tubulointerstitial nephritis [8] showed that tissue mRNA expression of Th2 (IL-4) and Treg 


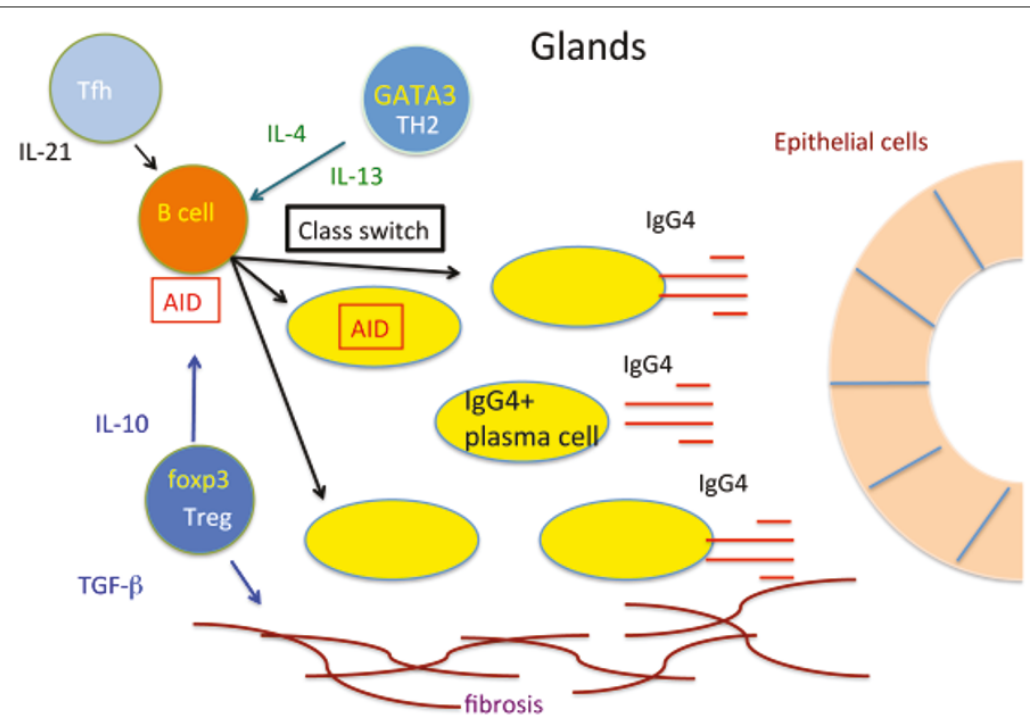

Figure 1. Molecular mechanism of IgG4-related disease. AID, activation-induced cytidine deaminase; IL, interleukin; TGF- $\beta$, transforming growth factor-beta; Th, T helper; Treg, regulatory T.

cytokines (IL-10 and TGF- $\beta$ ) was substantially higher than in other diseases. Many mononuclear cells expressing IL-4 or IL-10 were identifiable in affected organs by in situ hybridization [7]. Moreover, circulating CD4 ${ }^{+} \mathrm{CD} 25^{+}$Treg cells were significantly increased in PBMCs from patients with autoimmune pancreatitis [9].

Further examinations should shed light on the molecular mechanisms controlling the activation of this pathway.

\section{Abbreviations}

AID, activation-induced cytidine deaminase; APRIL, a proliferation-inducing ligand; BAFF, B cell-activating factor; IgG4-RD, IgG4-related disease; IL, interleukin; IRF4, interferon regulatory factor 4; LSG, labial salivary gland; MD, Mikulicz's disease; PBMC, peripheral blood mononuclear cell; SS, Sjögren's syndrome; TGF- $\beta$, transforming growth factor-beta; Th, T helper; Treg, regulatory $T$.

\section{Competing interests}

The author declares that he has no competing interests.

Published: 25 January 2013

\section{References}

1. Umehara H, Okazaki K, Masaki Y, Kawano M, Yamamoto M, Saeki T, Matsui S, Sumida T, Mimori T, Tanaka Y, Tsubota K, Yoshino T, Kawa S, Suzuki R, Takegami T, Tomosugi N, Kurose N, Ishigaki Y, Azumi A, Kojima M, Nakamura S, Inoue D: A novel clinical entity, IgG4-related disease (IgG4RD): general concept and details. Mod Rheumatol 2012, 22:1-14.
2. Stone JS, Yoh Z, Vikram D: IgG4-related disease. N Eng/ J Med 2012, 366:539-551.

3. Aalverse RC, Stapel SO, Schuurman J, Rispens T: Immunoglobulin G4: an odd antibody. Clin Exp Allergy 2009, 39:469-477.

4. Nirula A, Glaser SM, Kalled SL, Taylar FR: What is IgG4? A review of the biology of a unique immunoglobulin subtype. Curr Opin Rheumatol 2011, 23:119-124

5. Tsuboi H, Matsuo N, lizuka M, Tsuzuki S, Kondo Y, Tanaka A, Moriyama M, Matsumoto I, Nakamura S, Sumida T: Analysis of IgG4 class switch-related molecules in IgG4-related disease. Arthritis Res Ther 2012, 14:R171.

6. Tanaka A, Moriyama M, Nakashima H, Miyake K, Hayashida JN, Maehara T, Shinozaki S, Kubo Y, Nakamura S: Th2 and regulatory immune reactions contributes to lgG4 production and the initiation of Mikulicz's disease. Arthritis Rheum 2012, 64:254-263.

7. Zen Y, Fujii T, Harada K, Kawano M, Yamada K, Takahira M, Nakanuma Y: Th2 and regulatory immune reaction are increased in immunoglobin G4-related sclerosing pancreatitis and cholangitis. Hepatology 2007, 45:1538-1546.

8. Nakashima H, Miyake K, Moriyama M, Tanaka A, Watanabe M, Abe Y, Sato H, Nakamura S, Saito T: An amplification of IL-10 and TGF-beta in patients with IgG4-related tubulointerstitial nephritis. Clin Nephrol 2010, 73:385-391.

9. Miyoshi H, Uchida K, Taniguchi T, Yazumi S, Matsushita M, Takaoka M, Okazaki $\mathrm{K}$ : Circulating naïve and $\mathrm{CD} 4+\mathrm{CD} 25 \mathrm{high}$ regulatory $\mathrm{T}$ cells in patients with autoimmune pancreatitis. Pancreas 2008, 36:133-140.

doi:10.1186/ar4122

Cite this article as: Koike T: IgG4-related disease: why high IgG4 and fibrosis? Arthritis Research \& Therapy 2013, 15:103. 